\title{
Enlargement of filtrations and continuous Girsanov-type embeddings
}

\author{
Stefan Ankirchner ${ }^{1}$, Steffen Dereich ${ }^{2}$, and Peter Imkeller ${ }^{1}$ \\ 1 Institut für Mathematik \\ Humboldt-Universität zu Berlin \\ Unter den Linden 6 \\ 10099 Berlin \\ Germany \\ ankirch@math.tu-berlin.de imkeller@mathematik.hu-berlin.de \\ 2 Fachbereich Mathematik \\ Technische Universität Berlin \\ Strasse des 17. Juni 136 \\ 10623 Berlin \\ Germany \\ dereich@math.tu-berlin.de
}

Summary. Let $\left(\mathcal{G}_{t}\right)$ be an enlargement of the filtration $\left(\mathcal{F}_{t}\right)$. Jeulin and Jacod discussed a sufficient criterion for the inheritance of the semimartingale property when passing to the larger filtration. We provide alternative proofs of their results in a more general setting by using decoupling measures and Girsanov's changes of measure. We derive necessary and sufficient conditions for the embedding of vector spaces of $\left(\mathcal{F}_{t}\right)$-semimartingales into spaces of $\left(\mathcal{G}_{t}\right)$-semimartingales to be continuous in terms of generalized entropies of the information increment.

2000 AMS subject classifications: primary 60G44; secondary 60G48, 94A17, 62B10.

Key words and phrases: enlargement of filtration; semimartingale; decoupling measure; Girsanov's theorem; Shannon information; entropy.

\section{Introduction}

On a probability space, let $\left(\mathcal{G}_{t}\right)$ be a filtration containing a smaller filtration $\left(\mathcal{F}_{t}\right)$. The basic question of the well known theory of enlargement of filtrations (see [JY85]) with some relevance in simple models of financial markets with asymmetric information (see for instance [Imk03]) is this: under which conditions every $\left(\mathcal{F}_{t}\right)$-semimartingale remains a semimartingale relative to $\left(\mathcal{G}_{t}\right)$ ? In the pioneering papers of [JY85] this inheritance property has been called 'Hypothèse (H')'. Jacod [Jac85] gives a sufficient criterion for it to hold and studies semimartingales' Doob-Meyer decompositions relative to $\left(\mathcal{G}_{t}\right)$. With 
respect to vector space topologies on the set of $\left(\mathcal{F}_{t}\right)$ - and $\left(\mathcal{G}_{t}\right)$-semimartingales Yor [Yor85] investigates continuity properties of the associated mapping of $\left(\mathcal{F}_{t}\right)$-semimartingales into the space of $\left(\mathcal{G}_{t}\right)$-semimartingales.

In this paper we reconsider the problem of the inheritance of the semimartingale property from a different and more general perspective. In fact, in section 1 we derive inheritance results generalizing Jacod's [Jac85], which were proved in the setting of initial enlargements by the information stored in random elements with values in Lusin spaces. Our proofs are based on the concept of the decoupling measure, which allows an independent view on the additional information contained in the enlarged filtration, specified in $\sigma$-fields $\mathcal{H}_{t}$ enlarging $\mathcal{F}_{t}$ to obtain $\mathcal{G}_{t}=\mathcal{F}_{t} \vee \mathcal{H}_{t}$. The key observation is that under the decoupling measure every $\left(\mathcal{F}_{t}\right)$-martingale is a $\left(\mathcal{G}_{t}\right)$-martingale. Hence, enlarging the filtration can be seen as stepping from a view of processes through the decoupling measure to a view by the original measure. In particular, the associated Girsanov transform can be used to obtain explicit representations of the Doob-Meyer decomposition w.r.t. the larger filtration. This idea goes back to [FI93], where this method was used to analyze initial enlargements of the Wiener filtration by some random variable $G$. Later [AIS98] and [GP98] extended these techniques to more general stochastic bases and semimartingales. In more recent approaches it was rediscovered in terms of a Bayesian interpretation of simple models of insider trading by Gasbarra and Valkeila [GV03]. Of course, the cost of this approach consists in the very assumption of the existence of the decoupling measure. It restricts generality to a nontrivial extent, as is seen if compared for example to the setting of [ADI04]. For instance, if the information drift to be deducted from a martingale in the larger filtration does not generate an equivalent martingale measure capturing the change of views from the small to the large filtration, then there will be no decoupling measure. In order to tackle the problem, as Yoeurp [Yoe85] for the analysis of progressive enlargements, we choose a formulation a product space: the first marginal contains the original information, while the second describes the additional information. Under the product measure both marginals are independent. Therefore it will be the appropriate candidate for our decoupling measure.

Here is an outline of the structure of the material presented. Our main occupation in section 1 consists in showing how objects are transferred from the original space into the artificial product space and vice versa. Once this is handled, an application of the Girsanov transform leads to explicit DoobMeyer decompositions. In section 2 we provide estimates of the strength of the information drift by appropriate generalized entropies. These are used in section 3 in order to prove continuity properties of the embedding of the $\left(\mathcal{F}_{t}\right)$-semimartingales into the set of $\left(\mathcal{G}_{t}\right)$-semimartingales with respect to well known vector space topologies. These results generalize continuity results obtained by Yor [Yor85]. 


\section{Enlargement of filtrations and Girsanov's theorem}

Let $(\Omega, \mathcal{F}, P)$ be a probability space with right-continuous filtrations $\left(\mathcal{F}_{t}\right)_{t \geq 0}$ and $\left(\mathcal{H}_{t}\right)_{t \geq 0}$. Moreover, let $\mathcal{F}_{\infty}=\bigvee_{t \geq 0} \mathcal{F}_{t}$ and $\mathcal{H}_{\infty}=\bigvee_{t \geq 0} \mathcal{H}_{t}$.

Our objective is to study the enlarged filtration

$$
\mathcal{G}_{t}=\bigcap_{s>t}\left(\mathcal{F}_{s} \vee \mathcal{H}_{s}\right), \quad t \geq 0
$$

We relate this enlargement to a measure change on the product space

$$
\bar{\Omega}=\Omega \times \Omega
$$

equipped with the $\sigma$-field

$$
\overline{\mathcal{F}}=\mathcal{F}_{\infty} \otimes \mathcal{H}_{\infty}
$$

We endow $\bar{\Omega}$ with the filtration

$$
\overline{\mathcal{F}}_{t}=\bigcap_{s>t}\left(\mathcal{F}_{s} \otimes \mathcal{H}_{s}\right), \quad t \geq 0 .
$$

$\Omega$ will be embedded into $\bar{\Omega}$ by the map

$$
\psi:(\Omega, \mathcal{F}) \rightarrow(\bar{\Omega}, \overline{\mathcal{F}}), \omega \mapsto(\omega, \omega)
$$

We denote by $\bar{P}$ the image of the measure $P$ under $\psi$, i.e.

$$
\bar{P}=P_{\psi}
$$

Hence for all $\overline{\mathcal{F}}$-measurable functions $f: \bar{\Omega} \rightarrow \mathbb{R}$ we have

$$
\int f\left(\omega, \omega^{\prime}\right) d \bar{P}\left(\omega, \omega^{\prime}\right)=\int f(\omega, \omega) d P(\omega)
$$

In the following measure the two components in $\bar{\Omega}$ are decoupled, and weighted according to $P$ :

$$
\bar{Q}=\left.\left.P\right|_{\mathcal{F}_{\infty}} \otimes P\right|_{\mathcal{H}_{\infty}}
$$

We use notations and concepts of stochastic analysis as explained in the book by Protter [Pro04]. Our results will be stated for completed filtrations. We remark that due to our general assumption 1 below, all possible probability measures on the enlarged space we will consider possess systems of null sets that are at least bigger than the one related to $\bar{Q}$. So we could refer to the same completion throughout, and working with completions will not reduce generality. We shall use the following notation. Let $\left(\mathcal{K}_{t}\right)$ be a filtration and $R$ a probability measure. We denote by $\left(\mathcal{K}_{t}^{R}\right)$ the filtration $\left(\mathcal{K}_{t}\right)$ completed by the $R$-negligible sets.

The map $\psi$ will be used to translate processes $\bar{X}=\left(\bar{X}_{t}\right)_{t \geq 0}$ defined on $\left(\bar{\Omega}, \overline{\mathcal{F}}^{\bar{P}}\right)$ into processes $X=\bar{X} \circ \psi$ defined on $\left(\Omega, \mathcal{F}^{P}\right)$. The following Proposition shows that structural properties are preserved by this embedding. 
Proposition 1. Let $\bar{X}=\left(\bar{X}_{t}\right)_{t \in[0, \infty)}$ and $\bar{Y}$ denote stochastic processes and $\bar{T}$ a random time all defined on the measurable space $(\bar{\Omega}, \overline{\mathcal{F}} \bar{P})$. We set $X=\bar{X} \circ \psi$, $Y=\bar{Y} \circ \psi$ and $T=\bar{T} \circ \psi$. Then adaptedness, predictability, the local martingale or semimartingale properties are transferred from $\bar{X}$ with respect to $\left(\overline{\mathcal{F}}_{t}^{\bar{P}}\right)$ and $\bar{P}$ to $\bar{Y}$ with respect to $\left(\mathcal{G}_{t}^{P}\right)$ and $P$. If $\bar{T}$ is a $\left(\overline{\mathcal{F}}_{t}^{\bar{P}}\right)$-stopping time, then $T$ is a $\left(\mathcal{G}_{t}^{P}\right)$-stopping time. Moreover, if $\bar{X}$ is a $\left(\overline{\mathcal{F}}_{t}^{\bar{P}}, \bar{P}\right)$-semimartingale and $\bar{Y}$ is a càglàd $\left(\overline{\mathcal{F}}_{t}^{\bar{P}}, \bar{P}\right)$-adapted process, then

$$
\left(\int \bar{Y} d \bar{X}\right) \circ \psi=\int Y d X
$$

up to indistinguishability. If $\bar{X}$ and $\bar{Y}$ are $\left(\overline{\mathcal{F}}_{t}^{\bar{P}}, \bar{P}\right)$-semimartingales, then

$$
[\bar{X}, \bar{Y}] \circ \psi=[X, Y]
$$

up to indistinguishability.

Proof. Just observe that

$$
\begin{aligned}
\mathcal{G}_{t} & =\bigcap_{s>t} \sigma\left(A \cap B: A \in \mathcal{F}_{s}, B \in \mathcal{H}_{s}\right)=\bigcap_{s>t} \sigma\left(\psi^{-1}(A \times B): A \in \mathcal{F}_{s}, B \in \mathcal{H}_{s}\right) \\
& =\psi^{-1}\left(\bigcap_{s>t}\left(\mathcal{F}_{s} \otimes \mathcal{H}_{s}\right)\right)=\psi^{-1}\left(\overline{\mathcal{F}}_{t}\right)
\end{aligned}
$$

so that

$$
\psi^{-1}\left(\overline{\mathcal{F}}_{t}^{\bar{P}}\right) \subset \mathcal{G}_{t}^{P} .
$$

Now the properties follow by straightforward arguments.

In the reverse direction, structural properties are transferred quite as easily.

Lemma 1. Let $M$ be a right-continuous $\left(\mathcal{F}_{t}^{P}, P\right)$-local martingale. Then the process $\bar{M}\left(\omega, \omega^{\prime}\right)=M(\omega)$ is a $\left(\overline{\mathcal{F}}_{t}^{\bar{Q}}, \bar{Q}\right)$-local martingale.

Proof. Modulo localization, the martingale property follows readily by employing a monotone class argument to pass from indicators of rectangles to general bounded measurable functions in the smaller $\sigma$-field.

In the sequel we will always assume that $\bar{P}$ is absolutely continuous with respect to $\bar{Q}$, i.e.

\section{Assumption 1}

$$
\bar{P} \ll \bar{Q} \text { on } \overline{\mathcal{F}} \text {. }
$$

Note that this assumption is always satisfied if $\left(\mathcal{G}_{t}\right)$ is obtained by an initial enlargement by some discrete random variable $G$, i.e. $\mathcal{H}_{t}=\sigma(G)$ for all $t \geq 0$. In particular, this holds true for any progressive enlargement by $\mathcal{H}_{t}=\bigvee_{u \leq t}\{L \leq u\}$ where $L$ is discrete random time with values in $[0, \infty]$ (see also end of the section). 
Now let $M$ be a $\left(\mathcal{F}_{t}^{P}, P\right)$-local martingale and $\bar{M}$ its extension to $\bar{\Omega}$ as in Lemma 1. Since $\bar{P} \ll \bar{Q}, \bar{M}$ is a $\left(\overline{\mathcal{F}}_{t}^{\bar{P}}, \bar{P}\right)$-semimartingale and hence, by Proposition $1, M$ is a $\left(\mathcal{G}_{t}^{P}, P\right)$-semimartingale. Thus, clearly hypothesis $\left(\mathrm{H}^{\prime}\right)$ is satisfied. But what is its Doob-Meyer decomposition relative to $\left(\mathcal{G}_{t}^{P}, P\right)$ ?

Essentially the change of filtrations corresponds to changing the measure from $\bar{Q}$ to $\bar{P}$ on the product space $\bar{\Omega}$. Girsanov's theorem applies on $\bar{\Omega}$, since the measure $\bar{P}$ is absolutely continuous with respect to $\bar{Q}$. As a consequence we obtain a Girsanov-type result for the corresponding change of filtrations. For its explicit description we introduce the density process. Let $\left(\bar{Z}_{t}\right)$ denote a cadlag $\left(\overline{\mathcal{F}}_{t}^{\bar{Q}}, \bar{Q}\right)$-martingale satisfying

$$
\bar{Z}_{t}=\left.\frac{d \bar{P}}{d \bar{Q}}\right|_{\overline{\mathcal{F}}_{t}^{\bar{Q}}} .
$$

We are now in a position to state the main Girsanov-type result.

Theorem 1. If $M$ is a continuous $\left(\mathcal{F}_{t}^{P}, P\right)$-local martingale with $M_{0}=0$, then

$$
M-\frac{1}{Z_{-}} \cdot[M, Z]
$$

is a $\left(\mathcal{G}_{t}^{P}, P\right)$-local martingale.

Proof. Let $M$ be a continuous $\left(\mathcal{F}_{t}^{P}, P\right)$-local martingale with $M_{0}=0$. Lemma 1 implies that the process defined by $\bar{M}\left(\omega, \omega^{\prime}\right)=M(\omega)$ is a $\left(\overline{\mathcal{F}}_{t}^{\bar{Q}}\right)$-local martingale and the Girsanov Theorem (see for instance [Pro04], p. 136) yields that

$$
\bar{M}-\frac{1}{\bar{Z}_{-}} \cdot[\bar{M}, \bar{Z}]
$$

is a $\left(\overline{\mathcal{F}}_{t}^{\bar{P}}, \bar{P}\right)$-local martingale. It remains to appeal to simple transfer properties stated in Proposition 1.

Remark 1. Similar results as in Theorem 1 may of course be derived for noncontinuous martingales $M$ (see [Ank05] for details).

The preceding may also be formulated in terms of the stochastic logarithm of the density process $\bar{Z}$. To this end set $\bar{S}^{\prime}=\inf \left\{t>0: \bar{Z}_{t}=0\right\}$ and

$$
\bar{S}= \begin{cases}\bar{S}^{\prime} & \text { if } \bar{S}^{\prime}<\infty \text { and } \Delta \bar{Z}_{\bar{S}^{\prime}}=0 \\ \infty & \text { otherwise. }\end{cases}
$$

$\bar{S}$ is a $\left(\overline{\mathcal{F}}_{t}^{\bar{Q}}\right)$-predictable stopping time and we define

$$
\bar{L}=\int_{0+}^{\cdot} \frac{1}{\bar{Z}_{-}} d \bar{Z} \text { on }[0, \bar{S}[
$$

with the convention that $\bar{L}_{t}=\bar{L}_{\bar{S}^{\prime}}$ for $t \in\left[\bar{S}^{\prime}, \bar{S}[\right.$. So far, the process $\bar{L}$ is determined $\bar{P}$-, but not $\bar{Q}$-almost everywhere. (In order to define it everywhere we may put $\bar{L}=0$ on $\left[\bar{S}, \infty\left[\right.\right.$.) Then $\bar{L}$ is an $\left(\overline{\mathcal{F}}_{t}^{\bar{P}}, \bar{P}\right)$-semimartingale but 
not necessarily an $\left(\overline{\mathcal{F}}_{t}^{\bar{Q}}, \bar{Q}\right)$-semimartingale. However, restricted to the time interval $\left[0, \bar{S}\left[\right.\right.$ it is an $\left(\overline{\mathcal{F}}_{t}^{\bar{Q}}, \bar{Q}\right)$-local martingale. As usual we write $L=\bar{L} \circ \psi$. Alternatively, one can define $L$ through the stochastic integral $L=\int_{0+}^{\cdot} \frac{1}{Z_{-}} d Z$.

Since the process $\bar{L}$ is a $\left(\overline{\mathcal{F}}_{t}^{\bar{Q}}, \bar{Q}\right)$-local martingale on the interval $[0, \bar{S}[$, it can be decomposed into a unique continuous local-martingale part $\bar{L}^{c}$ starting in 0 and a sum of compensated jumps $\bar{L}^{d}$. As before, we consider the processes $L^{c}=\bar{L}^{c} \circ \psi$ and $L^{d}=\bar{L}^{d} \circ \psi$. Theorem 1 can now be reformulated as follows.

Theorem 2. If $M$ is a continuous $\left(\mathcal{F}_{t}^{P}, P\right)$-local martingale with $M_{0}=0$, then $M-[M, L]$ is a $\left(\mathcal{G}_{t}^{P}, P\right)$-local martingale.

Proof. The definition of $L$ implies that $\frac{1}{Z_{-}} \cdot[M, Z]=[M, L], P$-a.s. Now apply Theorem 1.

Finally, we will need the following formula, in which the subtracted drift is represented in terms of the quadration variation of the given local martingale.

Theorem 3. If $M$ is a continuous $\left(\mathcal{F}_{t}^{P}, P\right)$-local martingale with $M_{0}=0$, then there is a $\left(\mathcal{G}_{t}^{P}\right)$-predictable process $\alpha$, called information drift, such that $M-\alpha \cdot[M, M]$ is a $\left(\mathcal{G}_{t}^{P}\right)$-local martingale satisfying $P$-a.e.

$$
\int_{0}^{\infty} \alpha_{t}^{2} d[M, M]_{t} \leq[L, L]_{\infty}^{c}<\infty
$$

Proof. Let $M$ be a continuous $\left(\mathcal{F}_{t}^{P}, P\right)$-local martingale with $M_{0}=0$. As a consequence of the Kunita-Watanabe inequality (see for instance Lemme 1.36 in [Jac79] or page 136 of [Pro04]), there exists a $\left(\mathcal{G}_{t}^{P}\right)$-predictable process $\left(\alpha_{t}\right)$ such that

$$
\alpha \cdot[M, M]=[M, L]=\left[M, L^{c}\right] .
$$

The processes $M$ and $O=L^{c}-\alpha \cdot M$ are orthogonal w.r.t. $[\cdot, \cdot]$ so that

$$
\alpha^{2} \cdot[M, M]=[\alpha \cdot M, \alpha \cdot M] \leq\left[L^{c}, L^{c}\right]=[L, L]^{c} .
$$

Recall that $[L, L]=\left(\frac{1}{\bar{Z}_{-}^{2}} \cdot[\bar{Z}, \bar{Z}]\right) \circ \psi$ and that $\bar{Z}$ is a uniformly integrable nonnegative $\left(\overline{\mathcal{F}}_{t}^{\bar{Q}}, \bar{Q}\right)$-martingale. Since $\bar{P}$-a.s. $\bar{Z}_{\infty}>0$, one has also $\inf _{t \geq 0} \bar{Z}_{t}>0, \bar{P}$-a.s. Moreover, $[\bar{Z}, \bar{Z}]_{\infty}<\infty, \bar{Q}$-a.s. Therefore, $[\bar{L}, \bar{L}]$ is $\bar{P}$ a.s. bounded and consequently $[L, L]_{t}^{c}$ converges as $t \rightarrow \infty P$-a.s. to some real value which we denote by $[L, L]_{\infty}^{c}$.

Remark 2. Due to the previous theorem the information drift obtained via the Girsanov approach under Assumption 1 is always locally square integrable. It was shown in [ADI04] that in case $\Omega$ is standard Borel and each $\left(\mathcal{F}_{t}\right)$ martingale has a continuous modification, square integrability on the product space $\Omega \times[0, T]$ with respect to the measure $d[M, M] \otimes P$ implies the absolute continuity of the kernels $k_{t}\left(\cdot, d \omega^{\prime}\right)$ with respect to the conditional laws $P_{t}\left(\cdot, d \omega^{\prime}\right)$ of $\mathcal{G}_{t-}$ with respect to $\mathcal{F}_{t}$, where 


$$
P_{t}(\cdot, A)=P(A)+\int_{0}^{t} k_{s}(\cdot, A) d M_{s}+L_{t}^{A}, \quad A \in \mathcal{G}_{t-}, t \in[0, T],
$$

$L^{A}$ being orthogonal to $M$. In this case the R-N density process $\gamma_{t}\left(\omega, \omega^{\prime}\right)=$ $\frac{k_{t}\left(\cdot, d \omega^{\prime}\right)}{P_{t}\left(\cdot, d \omega^{\prime}\right)}$ is identical to $\alpha$ if restricted to the diagonal $\omega=\omega^{\prime}$. Hence this absolute continuity condition $(A C L)$ is implied by Assumption 1. Enlargements with locally integrable but not square integrable information drifts are beyond the scope of this article. But they provide examples for which $(A C L)$ does not imply Assumption 1. One example is obtained for instance by enlarging the Wiener filtration by the maximum of the Wiener process over some finite time interval. In this case Malliavin's calculus can be applied and an explicit representation of the information drift is obtained via the Clark-Ocone formula (see [IPW01] and [Imk03]). In case $\Omega$ is not standard Borel we do not know at the moment whether assumption 1 is more restrictive than $(A C L)$. The methods of [ADI04] allow in a more general setting the description of information drifts which are not necessarily locally square integrable.

\section{Comparison with Jacod's condition}

In Jacod's paper (see [Jac85]) the filtration $\left(\mathcal{F}_{t}\right)$ is supposed to be enlarged by some random variable $G$ taking values in a Lusin space $(E, \mathcal{E})$. As a consequence, for $t \in[0, T]$ regular conditional distributions $Q_{t}$ of $G$ relative to $\mathcal{F}_{t}$ exist. The following condition is assumed to be satisfied:

(A') For every $t \geq 0$ and $P$-a.a. $\omega$ the measure $Q_{t}(\omega, \cdot)$ is absolutely continuous with respect to the law $\eta$ of $G$.

We will show that in this setting condition $\left(\mathrm{A}^{\prime}\right)$ is equivalent to our assumption 1. More precisely, with $\mathcal{H}_{t}=\sigma(G)$, we have the following.

Lemma 2. (A') is satisfied if and only if $\bar{P} \ll \bar{Q}$ on $\overline{\mathcal{F}}_{t}$ for all $t \geq 0$.

Proof. First assume property (A'). Let $t \geq 0$ and $C \in \overline{\mathcal{F}}_{t}$ with $\bar{Q}(C)=0$. We choose $\tilde{C} \in \mathcal{F}_{t} \otimes \mathcal{E}$ such that

$$
1_{C}\left(\omega, \omega^{\prime}\right)=1_{\tilde{C}}\left(\omega, G\left(\omega^{\prime}\right)\right),
$$

and observe that

$$
\bar{Q}(C)=\int_{\bar{\Omega}} 1_{C}\left(\omega, \omega^{\prime}\right) d \bar{Q}\left(\omega, \omega^{\prime}\right)=\int_{\Omega}\left(\int_{E} 1_{\tilde{C}}(\omega, g) d \eta(g)\right) d P(\omega) .
$$

Hence for $P$-a.a. $\omega$ the set $C_{\omega}=\{g \in E:(\omega, g) \in \tilde{C}\}$ is a $\eta$-nullset. Consequently,

$$
\bar{P}(C)=\int_{\Omega} 1_{C}(\omega, \omega) d P(\omega)=\int Q_{t}\left(\omega, C_{\omega}\right) d P(\omega)
$$

is equal to 0 due to $\left(\mathrm{A}^{\prime}\right)$.

Now fix $t \geq 0$ and assume that $\bar{P} \ll \bar{Q}$ on $\overline{\mathcal{F}}_{s}$. Then there exists a $\overline{\mathcal{F}}_{t^{-}}$ measurable density $\varphi$ which can be represented in the form 


$$
\varphi(\omega, \tilde{\omega})=\tilde{\varphi}(\omega, G(\tilde{\omega}))
$$

where $\tilde{\varphi}$ is an appropriate $\mathcal{F}_{t} \otimes \mathcal{E}$-measurable function. Now integrating in $\tilde{\omega}$ will, by using Fubini's theorem in a similar manner as above, yield the conditional law of $G$ relative to $\mathcal{F}_{t}$ which is absolutely continuous with respect to $\eta$. This entails property $\left(\mathrm{A}^{\prime}\right)$.

Jacod does not use Girsanov's theorem in his paper [Jac85]. However, he points out that his results could also be deduced by applying it to the conditional measures $P^{x}=P(\cdot \mid G=x), x \in E$. Condition (A') implies that the conditional measures $P^{x}$ are absolutely continuous with respect to $P$. Hence, by Girsanov, for a given $\left(\mathcal{F}_{t}, P\right)$-local martingale there is a drift $A^{x}$ such that $M-A^{x}$ is a $\left(\mathcal{F}_{t}, P^{x}\right)$-local martingale. By combining the processes $A^{x}$ we obtain that

$$
M-A^{G}
$$

is a $\left(\mathcal{G}_{t}, P\right)$-local martingale. The main work consists in proving that the processes $A^{x}$ can be combined in a meaningful way. As far as we know, Jacod's sketch has never been worked out rigorously.

In our approach we embed every local martingale into the product space $\bar{\Omega}$. We apply Girsanov's theorem on the product space and then translate our results back into the original space. One of the advantages of our approach is that we do not have to assume regular conditional distributions to exist. And we do not need to show how processes can be combined. Instead we have to show how one can transfer objects from $\Omega$ to $\bar{\Omega}$ and vice versa. Moreover we are not restricted to initial enlargements, but only to enlargements of the form

$$
\mathcal{G}_{t}=\bigcap_{s>t}\left(\mathcal{F}_{s} \vee \mathcal{H}_{s}\right), \quad t \in[0, T]
$$

Starting with Jacod's results one can obtain decompositions for filtrations of this kind by using predictable projections. For this suppose $A$ to be a bounded variation process such that $M-A$ is a local martingale with respect to the initially enlarged filtration $\left(\mathcal{F}_{t} \vee \mathcal{H}_{\infty}\right)$. If $B$ is the predictable projection of $A$ onto $\left(\mathcal{G}_{t}\right)$, then $M-B$ is a $\left(\mathcal{G}_{t}\right)$-local martingale.

\section{Estimates for the drift}

Suppose $M$ is a continuous $\left(\mathcal{F}_{t}^{P}, P\right)$-local martingale with $M_{0}=0$. Under the assumptions of the previous section we know that there is a $\left(\mathcal{G}_{t}^{P}\right)$-predictable process $\alpha$ such that $M-\alpha \cdot[M, M]$ is $\left(\mathcal{G}_{t}^{P}, P\right)$-local martingale. Moreover, the information drift $\alpha$ satisfies

$$
\left(\alpha^{2} \cdot[M, M]\right)_{\infty} \leq[L, L]_{\infty}^{c} .
$$

In this section we provide bounds for

$$
E\left[\left(\alpha^{2} \cdot[M, M]\right)_{\infty}^{p}\right]
$$


for various moments $p \geq 1$ based on inequality (4).

Throughout this section we suppose the assumptions of the previous section and maintain the notation. More precisely, we assume that $\bar{P} \ll \bar{Q}$, denote by $\bar{Z}_{t}=\left.\frac{d \bar{P}}{d Q}\right|_{\overline{\mathcal{F}}_{t}^{\bar{Q}}}$ the density process, and by $\bar{L}$ the stochastic logarithm of $\bar{Z}$. We use again the decomposition of $\bar{L}$ into a continuous part $\bar{L}^{c}$ and a part $\bar{L}^{d}$ consisting of compensated jumps. As before we denote by $Z, L$ and $L^{c}$ the corresponding $\left(\mathcal{G}_{t}\right)$-adapted processes obtained by a right side application of $\psi$.

\subsection{Moment $p=1$}

Recall that the relative entropy of two probability measures $P$ and $Q$ on some $\sigma$-algebra $\mathcal{M}$ is defined by

$$
H_{\mathcal{M}}(P \| Q)= \begin{cases}E^{P}\left(\left.\log \frac{d P}{d Q}\right|_{\mathcal{M}}\right), & \text { if } P \ll Q \text { on } \mathcal{M} \\ \infty, & \text { if not } P \ll Q \text { on } \mathcal{M}\end{cases}
$$

In our situation, the relative entropy $H_{\overline{\mathcal{F}}}(\bar{P} \| \bar{Q})$ provides an upper bound for the first moment of $[L, L]^{c}$ :

\section{Lemma 3.}

$$
\frac{1}{2} E^{P}[L, L]_{\infty}^{c} \leq H_{\overline{\mathcal{F}}}(\bar{P} \| \bar{Q})
$$

If $\left(\bar{Z}_{t}\right)_{t \geq 0}$ is continuous and $\bar{Z}_{0}=1$, then one even has

$$
\frac{1}{2} E^{P}[L, L]_{\infty}=H_{\overline{\mathcal{F}}}(\bar{P} \| \bar{Q}) .
$$

Remark 3. If the $\sigma$-field $\mathcal{F}_{0}$ is trivial, then the measures $\bar{P}$ and $\bar{Q}$ coincide on $\mathcal{F}_{0} \otimes \mathcal{H}_{0}$, and hence in this case $\bar{Z}_{0}=1$.

Proof. Let $\left(\bar{T}_{n}\right)$ denote an increasing sequence of stopping times with $\lim _{n \rightarrow \infty} \bar{T}_{n} \geq$ $\bar{S}^{\prime}$. Since $\left(\bar{Z}_{t}\right)$ is a uniformly integrable $\left(\overline{\mathcal{F}}_{t}^{\bar{Q}}\right)$-martingale Jensen's inequality implies that

$$
E^{\bar{Q}} \bar{Z}_{\bar{T}_{n}} \log \bar{Z}_{\bar{T}_{n}} \leq E^{\bar{Q}} \bar{Z}_{\infty} \log \bar{Z}_{\infty}
$$

so that Fatou's lemma leads to

$$
\lim _{n \rightarrow \infty} E^{\bar{Q}} \bar{Z}_{\bar{T}_{n}} \log \bar{Z}_{\bar{T}_{n}}=E^{\bar{Q}} \bar{Z}_{\infty} \log \bar{Z}_{\infty}=H_{\overline{\mathcal{F}}}(\bar{P} \| \bar{Q}) .
$$

On $[0, \bar{S}[$ we decompose $\bar{L}$ into its continuous and discontinuous part $\bar{L}=$ $\bar{L}^{c}+\bar{L}^{d}$ and let $\bar{Z}_{t}^{c}=\mathcal{E}\left(\bar{L}^{c}\right)_{t}$ and $\bar{Z}_{t}^{d}=\bar{Z}_{0} \mathcal{E}\left(\bar{L}^{d}\right)_{t}$. Then $\bar{Z}_{t}=\bar{Z}_{t}^{c} \bar{Z}_{t}^{d}$ and

$$
\bar{Z}_{t} \log \bar{Z}_{t}=\bar{Z}_{t} \log \bar{Z}_{t}^{c}+\bar{Z}_{t} \log \bar{Z}_{t}^{d} .
$$

Now Itôs formula implies that $\bar{A}_{t}=\bar{Z}_{t} \log \bar{Z}_{t}^{d}$ is a $\left(\overline{\mathcal{F}}_{t}^{\bar{Q}}, \bar{Q}\right)$-local submartingale on $[0, \bar{S}[$. In fact, with $\xi(x)=x \log x$ for $x>0$ and $\xi(0)=0$ one obtains on $[0, \bar{S}[$ 


$$
\begin{aligned}
\bar{A}_{t}= & \xi\left(\bar{Z}_{0}\right)+\int_{0+}^{t} \xi\left(\bar{Z}_{s-}^{d}\right) d \bar{Z}_{s}^{c}+\int_{0+}^{t} \bar{Z}_{s-}^{c} \xi^{\prime}\left(\bar{Z}_{s-}^{d}\right) d \bar{Z}_{s}^{d} \\
& +\sum_{0<s \leq t} \bar{Z}_{s-}^{c}\left(\xi\left(\bar{Z}_{s}^{d}\right)-\xi\left(\bar{Z}_{s-}^{d}\right)-\xi^{\prime}\left(\bar{Z}_{s-}^{d}\right) \Delta \bar{Z}_{s}^{d}\right),
\end{aligned}
$$

where all summands in the previous line are non-negative due to the convexity of $\xi$.

Next, note that due to the Girsanov transform

$$
\bar{L}^{c}-[\bar{L}, \bar{L}]^{c}
$$

is a $\left(\overline{\mathcal{F}}_{t}^{\bar{Q}}, \bar{P}\right)$-local martingale. Now choose an increasing sequence of bounded stopping times $\left(\bar{T}_{n}\right)_{n \in \mathbb{N}}$ such that $\left(\bar{L}^{c}-[\bar{L}, \bar{L}]^{c}\right)^{\bar{T}_{n}}$ is a $\left(\overline{\mathcal{F}}_{t}^{\bar{Q}}, \bar{P}\right)$-martingale, $\bar{A}^{\bar{T}_{n}}$ is a $\bar{Q}$-submartingale and $\lim _{n \rightarrow \infty} \bar{T}_{n} \geq \bar{S}^{\prime}$. Such a sequence exists, and combining the above results gives

$$
\begin{aligned}
E^{\bar{Q}} \bar{Z}_{\bar{T}_{n}} \log \bar{Z}_{\bar{T}_{n}} \geq E^{\bar{Q}} \bar{Z}_{\bar{T}_{n}} \log \bar{Z}_{\bar{T}_{n}}^{c}+E^{\bar{Q}} \bar{Z}_{0} \log \bar{Z}_{0}^{c} \\
\\
\geq E^{\bar{P}} \log \bar{Z}_{\bar{T}_{n}}^{c}=\frac{1}{2} E^{\bar{P}}[\bar{L}, \bar{L}]_{\bar{T}_{n}}^{c} .
\end{aligned}
$$

The first assertion follows by equation (5).

If $\bar{Z}$ is continuous and $\bar{Z}_{0}=1$, then $\bar{Z}_{t}=\bar{Z}_{t}^{c}$ which implies that

$$
E^{\bar{Q}} \bar{Z}_{\bar{T}_{n}} \log \bar{Z}_{\bar{T}_{n}}=\frac{1}{2} E^{\bar{P}}[\bar{L}, \bar{L}]_{\bar{T}_{n}}^{c} .
$$

The second assertion is an immediate consequence of equation (5).

\subsection{Moments $p>1$}

Now we consider moments of order $p>1$. In this case the $p$-th moment of $[L, L]_{\infty}$ can be compared to some generalized relative entropy. See [Imk96] for elementary versions of the inequalities to be derived.

Our analysis requires some additional assumption. We suppose that $\left(\mathcal{G}_{t}\right)$ is an initial enlargement of $\left(\mathcal{F}_{t}\right)$, i.e.

$$
\mathcal{G}_{t}=\bigcap_{s>t}\left(\mathcal{F}_{s} \vee \mathcal{A}\right), \quad t \geq 0
$$

where $\mathcal{A}$ is some fixed sub- $\sigma$-algebra of $\mathcal{F}$. Moreover, we assume that $\mathcal{F}_{0}$ is trivial. As in [Yor85], we need to impose the following additional assumption.

Assumption 2 (C) Every $\left(\mathcal{F}_{t}^{P}, P\right)$-martingale has a continuous modification.

We shall see that under this condition $\bar{L}$ is a continuous $\left(\overline{\mathcal{F}}_{t}^{\bar{Q}}, \bar{Q}\right)$-local martingale. We begin with the definition of the generalized relative entropy.

Definition 1. For $p>1$, and probability measures $P \ll Q$ on a $\sigma$-algebra $\mathcal{M}$, let

$$
H_{\mathcal{M}}^{p}(P \| Q):=E^{P}\left(\left.\log _{+} \frac{d P}{d Q}\right|_{\mathcal{M}}\right)^{p}
$$


We provide now an upper bound of $E[L, L]_{\infty}^{p}$ with the help of the generalized entropy of $\bar{P}$ with respect to $\bar{Q}$ on the set $\overline{\mathcal{F}}_{\infty}$. To simplify notations, we omit the $\sigma$-algebra $\overline{\mathcal{F}}_{\infty}$, and write only $H^{p}(\bar{P} \| \bar{Q})$ and $H(\bar{P} \| \bar{Q})$. The aim of this section is to prove

Theorem 4. For any $p \geq 1$ there exists a universal constant $C=C(p)<\infty$ such that under the above assumptions one has

$$
E[L, L]_{\infty}^{p} \leq C\left[H(\bar{P} \| \bar{Q})+H^{p}(\bar{P} \| \bar{Q})\right] .
$$

For the proof we need some auxiliary results. We start by showing that there exists a continuous modification for $\bar{Z}$.

Lemma 4. Let $\bar{M}$ be a uniformly integrable $\left(\overline{\mathcal{F}}_{t}^{\bar{Q}}, \bar{Q}\right)$-local martingale. If assumption $(C)$ is satisfied, then for P-a.a. $\omega^{\prime}$ the process $\bar{M}^{\omega^{\prime}}=\bar{M}\left(\cdot, \omega^{\prime}\right)$ is a $\left(\mathcal{F}_{t}^{P}\right)$-local martingale.

Proof. Choose a modification such that every path of $\bar{M}$ is cadlag. Now let $\hat{M}$ be an $\mathcal{A} \otimes \mathcal{O}(\mathcal{F})$-measurable process such that for all $\omega^{\prime}$ and $s \geq 0$

$$
\hat{M}_{s}^{\omega^{\prime}}=E^{P}\left[\bar{M}_{\infty}^{\omega^{\prime}} \mid \mathcal{F}_{s}\right] .
$$

For the existence of such a process we refer to [SY78], Proposition 3. Put $C_{t}=\left\{\hat{M}_{t}>\bar{M}_{t}\right\}$. Clearly $C_{t} \in \overline{\mathcal{F}}_{t}^{\bar{Q}}$ and $C_{t}\left(\cdot, \omega^{\prime}\right) \in \mathcal{F}_{t}^{P}$ for all $P$-a.a. $\omega^{\prime}$ (recall that $\left(\mathcal{F}_{t}\right)$ is right-continuous). Moreover for $t \geq 0$

$$
\begin{aligned}
& \iint 1_{C_{t}}\left(\omega, \omega^{\prime}\right)\left(\hat{M}_{t}^{\omega^{\prime}}-\bar{M}_{t}^{\omega^{\prime}}\right) d P(\omega) d P\left(\omega^{\prime}\right) \\
= & E^{\bar{Q}}\left[1_{C_{t}}\left(\hat{M}_{t}-\bar{M}_{t}\right)\right] \\
= & E^{\bar{Q}}\left[1_{C_{t}}\left(\hat{M}_{t}-\bar{M}_{\infty}\right)\right] \\
= & \iint 1_{C_{t}}\left(\omega, \omega^{\prime}\right)\left(\hat{M}_{t}^{\omega^{\prime}}-\bar{M}_{\infty}^{\omega^{\prime}}\right) d P(\omega) d P\left(\omega^{\prime}\right) \\
= & \int 0 d P\left(\omega^{\prime}\right)=0,
\end{aligned}
$$

A similar result holds true on the set $\left\{\hat{M}_{t}<\bar{M}_{t}\right\}$, and as a consequence we have for $P$-a.a. $\omega^{\prime}$

$$
\hat{M}_{t}\left(\cdot, \omega^{\prime}\right)=\bar{M}_{t}\left(\cdot, \omega^{\prime}\right), P \text {-a.s. }
$$

Hence for $P$-a.a. $\omega^{\prime}$ the process $\left(\bar{M}_{q}^{\omega^{\prime}}\right)_{q \in \mathbb{Q}^{+}}$is a $\left(\mathcal{F}_{t}^{P}\right)$-martingale. Since $\bar{M}_{t}$ is cadlag and uniformly integrable we obtain that also

$$
\left(\bar{M}_{t}^{\omega^{\prime}}\right)_{t \geq 0}
$$

is a $\left(\mathcal{F}_{t}^{P}\right)$-martingale for $P$-a.a. $\omega^{\prime}$.

Lemma 5. If $(C)$ is satisfied, then every uniformly integrable $\left(\overline{\mathcal{F}}_{t}^{\bar{Q}}, \bar{Q}\right)$-local martingale has a continuous modification. 
Proof. Let $\bar{M}$ be a $\left(\overline{\mathcal{F}}_{t}^{\bar{Q}}, \bar{Q}\right)$-local martingale. We may suppose that $\bar{M}$ is cadlag everywhere, and hence, the set

$$
N=\left\{\left(\omega, \omega^{\prime}\right): t \mapsto \bar{M}_{t}\left(\omega, \omega^{\prime}\right) \text { is not continuous }\right\}
$$

is measurable. Fix $\omega^{\prime}$ and suppose that $\bar{M}^{\omega^{\prime}}$ is a $\left(\mathcal{F}_{t}^{P}\right)$-martingale. Then assumption (C) implies that for $P$-a.a. $\omega$ the paths $t \mapsto \bar{M}_{t}^{\omega^{\prime}}(\omega)$ are continuous, i.e. $P\left(N^{\omega^{\prime}}\right)=0$. Now Fubini's theorem yields with Lemma 4

$$
\begin{aligned}
E^{\bar{Q}}(N) & =\iint 1_{N \omega^{\prime}}(\omega) d P(\omega) d P\left(\omega^{\prime}\right) \\
& =\int 0 d P\left(\omega^{\prime}\right)=0,
\end{aligned}
$$

and hence the result.

For the rest of the section we will suppose that $\bar{Z}$ is a continuous modification of our density process $\left.\frac{d \bar{P}}{d \bar{Q}}\right|_{\overline{\mathcal{F}}_{t}^{\bar{Q}}}$. Similarly, $\bar{L}$ will be assumed to be continuous.

Proof (of Theorem 4). We assume that $H(\bar{P} \| \bar{Q})$ and $H^{p}(\bar{P} \| \bar{Q})$ are finite. Then $X_{t}:=\bar{L}_{t}-[\bar{L}, \bar{L}]_{t}$ is a continuous $L^{2}$-bounded $\bar{P}$-martingale by Lemma 3 and we write $\log \bar{Z}_{t}=X_{t}+\frac{1}{2} A_{t}$ with $A_{t}:=[\bar{L}, \bar{L}]_{t}=[X, X]_{t}$. Next, observe that

$$
\begin{aligned}
H^{p}(\bar{P} \| \bar{Q})^{1 / p} & =E^{\bar{P}}\left[\left(X_{\infty}+\frac{1}{2} A_{\infty}\right)_{+}^{p}\right]^{1 / p} \\
& \geq E^{\bar{P}}\left[\left(\frac{1}{2} A_{\infty}-\left(\left|X_{\infty}\right| \wedge \frac{1}{2} A_{\infty}\right)\right)^{p}\right]^{1 / p} \\
& \geq \frac{1}{2} E^{\bar{P}}\left[A_{\infty}^{p}\right]^{1 / p}-E^{\bar{P}}\left[\left|X_{\infty}\right|^{p}\right]^{1 / p} \\
& \geq \frac{1}{2} E^{\bar{P}}\left[A_{\infty}^{p}\right]^{1 / p}-C E^{\bar{P}}\left[A_{\infty}^{p / 2}\right]^{1 / p}
\end{aligned}
$$

where the last inequality holds for some constant $C>0$ due to the BurkholderDavis-Gundy inequality. Now choose $\xi>0$ such that for all $x \geq 0$

$$
C^{p} x^{p / 2} \leq \xi^{p} x+\frac{1}{4^{p}} x^{p} .
$$

This leads to

$$
C^{p} E^{\bar{P}} A_{\infty}^{p / 2} \leq \xi^{p} E^{\bar{P}} A_{\infty}+\frac{1}{4^{p}} E^{\bar{P}} A_{\infty}^{p}
$$

and hence to

$$
C E^{\bar{P}}\left[A_{\infty}^{p / 2}\right]^{1 / p} \leq \xi E^{\bar{P}}\left[A_{\infty}\right]^{1 / p}+\frac{1}{4} E^{\bar{P}}\left[A_{\infty}^{p}\right]^{1 / p} .
$$

With (6) we conclude that

$$
H^{p}(\bar{P} \| \bar{Q})^{1 / p} \geq \frac{1}{4} E^{\bar{P}}\left[A_{\infty}^{p}\right]^{1 / p}-\xi E^{\bar{P}}\left[A_{\infty}\right]^{1 / p}=\frac{1}{4} E^{\bar{P}}\left[A_{\infty}^{p}\right]^{1 / p}-\xi H(\bar{P} \| \bar{Q})^{1 / p}
$$

Consequently, 


$$
\begin{aligned}
E^{\bar{P}}\left[A_{\infty}^{p}\right]^{1 / p} & \leq 4 \xi H(\bar{P} \| \bar{Q})^{1 / p}+4 H^{p}(\bar{P} \| \bar{Q})^{1 / p} \\
& \leq 8\left(\xi^{p} H(\bar{P} \| \bar{Q})+H^{p}(\bar{P} \| \bar{Q})\right)^{1 / p}
\end{aligned}
$$

where the last step follows from the elementary inequality $a+b \leq 2\left(a^{p}+b^{p}\right)^{1 / p}$, $a, b \geq 0$.

Remark 4. The above proof is based on the fact that there exists a constant $C_{p}$ such that for any continuous $L^{2}$-bounded $\bar{P}$-martingale $\left(X_{t}\right)$ with $X_{0}=0$ and quadratic variation process $\left(A_{t}\right)$ one has

$$
E^{\bar{P}} A_{\infty}^{p} \leq C_{p} E^{\bar{P}}\left[X_{\infty}+\frac{1}{2} A_{\infty}+\left(X_{\infty}+\frac{1}{2} A_{\infty}\right)_{+}^{p}\right] .
$$

Improving the estimate to

$$
E^{\bar{P}} A_{\infty}^{p} \leq C_{p} E^{\bar{P}}\left(X_{\infty}+\frac{1}{2} A_{\infty}\right)_{+}^{p}
$$

would lead to the better estimate $E^{P}[L, L]^{p} \leq C_{p} H^{p}(\bar{P} \| \bar{Q})$. However, an estimate stating (7) is not valid, as the following example shows.

Example 1. Let $W$ be a Wiener process and for fixed $\varepsilon>0$, let $T$ denote the first hitting time of the slope $t \mapsto \varepsilon-t / 2$. We consider $X_{t}:=W_{t}^{T}$ and $A_{t}:=[X, X]_{t}$. Then by the Lévy-Bachelier formula the law of $T=A_{\infty}$ has density

$$
1_{(0, \infty)}(t) \frac{\varepsilon}{t^{3 / 2}} \phi\left(\frac{\varepsilon-t / 2}{\sqrt{t}}\right),
$$

where $\phi$ is the density of the standard normal law. Hence,

$$
E\left[A_{\infty}^{p}\right]=\varepsilon \int_{0}^{\infty} t^{p-3 / 2} \phi\left(\frac{\varepsilon-t / 2}{\sqrt{t}}\right) d t .
$$

In particular, for $\varepsilon \downarrow 0$, one has $E\left[A_{\infty}^{p}\right] \approx \varepsilon$. On the other hand,

$$
E\left[\left(X_{\infty}+\frac{1}{2} A_{\infty}\right)_{+}^{p}\right]=E\left[\left(W_{T}+T / 2\right)^{p}\right]=\varepsilon^{p}
$$

such that one can always find a sufficiently small $\varepsilon>0$ for which the inequality (7) is not valid.

We next show a result which in a sense contains the inverse statement to Theorem 4.

Lemma 6. For $p \geq 1$ there exists a universal constant $C=C(p)<\infty$ such that

$$
H^{p}(\bar{P} \| \bar{Q}) \leq C\left[E^{\bar{P}}[\bar{L}, \bar{L}]_{\infty}^{p}+1\right] .
$$

In particular finiteness of $E^{\bar{P}}\left([\bar{L}, \bar{L}]_{\infty}^{p}\right)$ implies finiteness of the entropy $H^{p}(\bar{P} \| \bar{Q})$. 
Proof. We have, by Burkholder-Davis-Gundy, with a universal constant $C_{1}$

$$
\begin{aligned}
H^{p}(\bar{P} \| \bar{Q})^{1 / p} & \leq E\left(\left|\bar{L}_{\infty}-\frac{1}{2}[\bar{L}, \bar{L}]_{\infty}\right|^{p}\right)^{1 / p} \\
& \leq E\left(\left|\bar{L}_{\infty}\right|^{p}\right)^{1 / p}+E\left(\frac{1}{2}[\bar{L}, \bar{L}]_{\infty}^{p}\right)^{1 / p} \\
& \leq C_{1} E\left([\bar{L}, \bar{L}]_{\infty}^{p / 2}\right)^{1 / p}+E\left(\frac{1}{2}[\bar{L}, \bar{L}]_{\infty}^{p}\right)^{1 / p} \\
& \leq C_{1}\left(1+E[\bar{L}, \bar{L}]_{\infty}^{p}\right)^{1 / p}+E\left(\frac{1}{2}[\bar{L}, \bar{L}]_{\infty}^{p}\right)^{1 / p} \\
& \leq C_{2}\left(1+E\left([\bar{L}, \bar{L}]_{\infty}^{p}\right)\right)^{1 / p}
\end{aligned}
$$

and thus the result.

Suppose now that the enlargement $\mathcal{A}$ is induced by some discrete random variable $G$, i.e. $\mathcal{A}=\sigma(G)$. In that case one can estimate the moments of $[L, L]_{\infty}$ against some generalized absolute entropy of $G$.

Definition 2. Let $\left(q_{g}\right)$ denote the probability weights of $G$. We denote by

$$
H^{p}(G)=\sum_{g} q_{g}\left(\log 1 / q_{g}\right)^{p} .
$$

the generalized absolute entropy of order $p$.

Lemma 7. One has

$$
H^{p}(\bar{P} \| \bar{Q}) \leq H^{p}(G),
$$

and if $G$ is $\mathcal{F}_{\infty}$-measurable, then

$$
H^{p}(\bar{P} \| \bar{Q})=H^{p}(G) .
$$

Proof. For the proof we need a monotonicity property of $f$-divergences. Due to Corollary 1.29 in [LV87] one has

$$
\begin{aligned}
H^{p}(\bar{P} \| \bar{Q}) & =H^{p}\left(P_{\mathrm{id}_{\mathcal{F}_{\infty}}, \operatorname{id}_{\mathcal{A}}} \| P_{\mathrm{id}_{\mathcal{F}_{\infty}}} \otimes P_{\mathrm{id}_{\mathcal{A}}}\right) \\
& \leq H^{p}\left(P_{\mathrm{id}_{\mathcal{F}_{\infty}}, G, \mathrm{id}_{\mathcal{A}}} \| P_{\mathrm{id}_{\mathcal{F}_{\infty}}, G} \otimes P_{\mathrm{id}_{\mathcal{A}}}\right) .
\end{aligned}
$$

Moreover, if $G$ is $\mathcal{F}_{\infty}$-measurable, then one even has equality in the previous line. We denote by $\left(q_{g}\right)$ the probability weights of $G$. One easily verifies that

$$
\frac{d P_{\operatorname{id}_{\mathcal{F}_{\infty}}, G, \mathrm{id}_{\mathcal{A}}}}{d P_{\operatorname{id}_{\mathcal{F}_{\infty}}, G} \otimes P_{\operatorname{id}_{\mathcal{A}}}}\left(\omega, g, \omega^{\prime}\right)=1_{\left\{g=G\left(\omega^{\prime}\right)\right\}} \frac{1}{q_{g}} .
$$

Set $f\left(g, g^{\prime}\right)=1_{\left\{g=g^{\prime}\right\}} \frac{1}{q_{g}}$. Then

$$
\begin{aligned}
& H^{p}\left(P_{\mathrm{id}_{\mathcal{F}_{\infty}}, G, \mathrm{id}_{\mathcal{A}}} \| P_{\left.\mathrm{id}_{\mathcal{F}_{\infty}, G} \otimes P_{\mathrm{id}_{\mathcal{A}}}\right)}\right. \\
= & \int f\left(g, G\left(\omega^{\prime}\right)\right)\left(\log _{+} f\left(g, G\left(\omega^{\prime}\right)\right)\right)^{p} d\left(P_{\mathrm{id}_{\mathcal{F}_{\infty}, G}} \otimes P_{\mathrm{id}_{\mathcal{A}}}\right)\left(\omega, g, \omega^{\prime}\right) \\
= & \int_{\left\{\left(g, \omega^{\prime}\right): g=G\left(\omega^{\prime}\right)\right\}} \frac{1}{q_{g}}\left(\log _{+} \frac{1}{q_{g}}\right)^{p} d\left(P_{G} \otimes P_{\operatorname{id}_{\mathcal{A}}}\right)\left(g, \omega^{\prime}\right),
\end{aligned}
$$


since $f\left(g, G\left(\omega^{\prime}\right)\right)=0$ if $g \neq G\left(\omega^{\prime}\right)$ and the integrand does not depend on $\omega$. Altogether, we arrive at

$$
H^{p}(\bar{P} \| \bar{Q}) \leq \sum_{g} q_{g}\left(\log \frac{1}{q_{g}}\right)^{p}=H^{p}(G)
$$

and equality holds if $G$ is $\mathcal{F}_{\infty}$-measurable.

Example 2. Let $M_{t}=W_{t}$ denote a Wiener process and consider the completed filtration $\left(\mathcal{F}_{t}\right)=\left(\mathcal{F}_{t}^{W}\right)$ generated by the Wiener process. We now consider an initial enlargement of the filtration $\left(\mathcal{F}_{t}\right)$ by some arbitrary $\sigma$-field $\mathcal{A}$, i.e. $\mathcal{G}_{t}=\bigcap_{s>t}\left(\mathcal{F}_{s} \wedge \mathcal{A}\right)$. Supposing that $\bar{P} \ll \bar{Q}$, the Doob-Meyer decomposition for $W$ with respect to $\left(\mathcal{G}_{t}\right)$ is of the form

$$
W_{t}=\tilde{W}_{t}+\int_{0}^{t} \alpha_{s} d s
$$

where $\tilde{W}$ is a $\left(\mathcal{G}_{t}\right)$-Wiener process and $\alpha$ is a $\left(\mathcal{G}_{t}\right)$-adapted process. In fact, $\tilde{W}$ is continuous with quadratic variation process $[\tilde{W}, \tilde{W}]_{t}=t$. Moreover, since $\mathcal{F}_{0}$ is trivial and all $\left(\mathcal{F}_{t}\right)$-martingales have continuous modifications, the results of this section lead to the estimate

$$
E\left(\int_{0}^{t} \alpha_{s}^{2} d s\right)^{p} \leq C_{p}\left[H(\bar{P} \| \bar{Q})+H^{p}(\bar{P} \| \bar{Q})\right] .
$$

If in addition $\mathcal{A}=\sigma(G)$ is generated by some discrete random variable $G$, then

$$
E\left(\int_{0}^{t} \alpha_{s}^{2} d s\right)^{p} \leq C_{p}\left[H(G)+H^{p}(G)\right] .
$$

\section{Continuity of initial enlargements}

In section 1 we have seen that every $\left(\mathcal{F}_{t}^{P}\right)$-semimartingale is also a semimartingale relative to a bigger filtration $\left(\mathcal{G}_{t}^{P}\right)$ if the measure $\bar{P}$ is absolutely continuous with respect to $\bar{Q}$. In this section we analyze to which extent this embedding of $\left(\mathcal{F}_{t}^{P}\right)$-semimartingales into some space of $\left(\mathcal{G}_{t}^{P}\right)$-semimartingales is continuous. For simplicity we restrict to initial enlargements. It turns out that the embedding is continuous if and only if some generalized entropy of the measures $\bar{P}$ and $\bar{Q}$ is finite.

Let $\left(\Omega, \mathcal{F},\left(\mathcal{F}_{t}\right), P\right)$ be a filtered probability space as in the previous section. Throughout this section we assume that $\mathcal{F}_{0}$ is trivial and we let

$$
\mathcal{G}_{t}=\bigcap_{s>t}\left(\mathcal{F}_{s} \vee \mathcal{A}\right), \quad t \geq 0,
$$

where $\mathcal{A}$ is some fixed sub- $\sigma$-algebra of $\mathcal{F}$. The measures $\bar{P}$ and $\bar{Q}$ are defined as in the previous section and we assume again that $\bar{P}$ is absolutely continuous with respect to $\bar{Q}$. As before we will abbreviate $\bar{Z}_{t}=\left.\frac{d \bar{P}}{d \bar{Q}}\right|_{\overline{\mathcal{F}}_{t}}, \quad t \geq 0$. For a treatment of basic questions and ideas of this section in the setting of initial enlargements by random variables see [Imk96]. 


\subsection{Preliminaries}

We now recall the definition of some basic norms on the set of semimartingales. For this let $X$ be a $\left(\mathcal{F}_{t}^{P}\right)$-semimartingale. Given a decomposition $X=M+A$ we define for all $1 \leq p<\infty$,

$$
j_{p}(M, A)=\left\|[M, M]_{\infty}^{\frac{1}{2}}+\int_{[0, \infty[}\left|d A_{s}\right|\right\|_{L^{p}}
$$

and

$$
\|X\|_{\mathcal{S}^{p}}=\inf _{X=M+A} j_{p}(M, A) .
$$

We denote by $\mathcal{S}^{p}$ the set of all $\left(\mathcal{F}_{t}^{P}\right)$-semimartingales $X$ such that $\|X\|_{\mathcal{S}^{p}}<\infty$. If we want to emphasize the filtration we are referring to we write $\mathcal{S}^{p}\left(\mathcal{F}_{t}\right)$. The space $\mathcal{S}^{p}$ is a Banach space with the following properties (see e.g. [DM82]):

- Any $X \in \mathcal{S}^{p}$ is a special semimartingale.

- Let $X \in \mathcal{S}^{p}$ and $X=\bar{M}+\bar{A}$ be the unique decomposition such that $\bar{A}$ is predictable and $\bar{A}_{0}=0$. There is a constant $c>0$, depending only on $p$, such that $j_{p}(\bar{M}, \bar{A}) \leq c\|X\|_{\mathcal{S}^{p}}$.

- The space of all martingales in $\mathcal{S}^{p}$, denoted by $\mathcal{H}^{p}$, is a closed subspace.

- The set of all continuous semimartingales in $\mathcal{S}^{p}$, denoted by $\mathcal{S}_{c}^{p}$, and the set of all continuous martingales in $\mathcal{S}^{p}$, denoted by $\mathcal{H}_{c}^{p}$, are closed subspaces.

- The set of all predictable processes with integrable variation, vanishing in 0 and with norm $A \mapsto\left\|\int\left|d A_{s}\right|\right\|_{L^{p}}$ is a closed subspace of $\mathcal{S}^{p}$.

We will see that under suitable conditions every semimartingale in $\mathcal{S}^{2}\left(\mathcal{F}_{t}\right)$ belongs to $\mathcal{S}^{1}\left(\mathcal{G}_{t}\right)$.

\subsection{Continuity and relative entropy}

We are now in a position to prove the first main result.

Theorem 5. Suppose $H(\bar{P} \| \bar{Q})=C<\infty$. Then the embedding

$$
\mathcal{H}_{c}^{2}\left(\mathcal{F}_{t}\right) \rightarrow \mathcal{S}^{1}\left(\mathcal{G}_{t}\right), X \mapsto X,
$$

is a continuous linear mapping with norm $\leq 1+\sqrt{2 C}$.

Proof. Let $M \in \mathcal{H}^{2}\left(\mathcal{F}_{t}\right)$. By Theorem $2,(M-[M, L])+[M, L]$ is a decomposition relative to $\left(\mathcal{G}_{t}\right)$. The Kunita-Watanabe inequality implies

$$
\left\|\int_{0}^{\infty}\left|d[M, L]_{t}\right|\right\|_{1} \leq\left\|[L, L]_{\infty}^{\frac{1}{2}}\right\|_{2}\left\|[M, M]_{\infty}^{\frac{1}{2}}\right\|_{2} .
$$

Hence by Lemma 3

$$
\begin{aligned}
\|M\|_{\mathcal{S}^{1}\left(\mathcal{G}_{t}\right)} & \leq\left\|[M, M]_{\infty}^{\frac{1}{2}}+\int_{0}^{\infty} \mid d[M, L]_{t}\right\|_{1} \\
& \leq\left(1+\left\|[L, L]_{\infty}^{\frac{1}{2}}\right\|_{2}\right)\left\|[M, M]_{\infty}^{\frac{1}{2}}\right\|_{2} \\
& \leq\left(1+\left(E[L, L]_{\infty}\right)^{\frac{1}{2}}\right)\|M\|_{\mathcal{H}^{2}\left(\mathcal{F}_{t}\right)} \\
& \leq(1+\sqrt{2 C})\|M\|_{\mathcal{H}^{2}\left(\mathcal{F}_{t}\right)},
\end{aligned}
$$


and the proof is complete.

As an immediate consequence we get the following

Corollary 1. Suppose $H(\bar{P} \| \bar{Q})<\infty$. Then the embedding

$$
\mathcal{S}_{c}^{2}\left(\mathcal{F}_{t}\right) \rightarrow \mathcal{S}^{1}\left(\mathcal{G}_{t}\right), X \mapsto X
$$

is a continuous linear mapping.

\subsection{Continuity and generalized entropy}

We aim at generalizing Theorem 5 and Corollary 1. Starting from the Banach space $\mathcal{S}^{r}\left(\mathcal{F}_{t}\right)$ with $r>1$, what are sufficient criteria for the embedding into the space of $\left(\mathcal{G}_{t}\right)$-semimartingales to be continuous?

Throughout this section we assume assumption (C). In other words, we will assume that $\mathcal{H}_{c}^{p}\left(\mathcal{F}_{t}\right)=\mathcal{H}^{p}\left(\mathcal{F}_{t}\right)$ for $p>1$.

We begin by stating a result obtained by Yor.

Lemma 8. (see Lemme 2 in [Yor85]) Let $r \geq 1$ and $p, q>0$ such that $\frac{1}{r}=$ $\frac{1}{2 p}+\frac{1}{q}$. Then the following conditions are equivalent:

1) There is a constant $C>0$ such that every continuous $\left(\mathcal{G}_{t}\right)$-local martingale satisfies

$$
\left\|\int_{0}^{\infty}\left|d[M, L]_{t}\right|\right\|_{r} \leq C\left\|[M, M]_{\infty}^{\frac{1}{2}}\right\|_{q}
$$

2) $E\left[[L, L]_{\infty}^{p}\right]<\infty$.

We are now ready to state the main theorem.

Theorem 6. Suppose assumption $(C)$ is satisfied and let $p \geq 1$ and $q, r \geq 0$ such that $\frac{1}{r}=\frac{1}{2 p}+\frac{1}{q}$. The generalized entropy $H^{p}(\bar{P} \| \bar{Q})$ is finite if and only if the embedding

$$
\mathcal{S}^{q}\left(\mathcal{F}_{t}\right) \rightarrow \mathcal{S}^{r}\left(\mathcal{G}_{t}\right), X \mapsto X
$$

is a continuous linear mapping.

Proof. Suppose $H^{p}(\bar{P} \| \bar{Q})<\infty$. Theorem 4 implies that $[L, L]_{\infty}$ is $L^{p}$ integrable. Thus, by Lemma 8 , there is a constant $C>0$ such that for all continuous $\left(\mathcal{G}_{t}\right)$-local martingales we have

$$
\left\|\int_{0}^{\infty}\left|d[M, L]_{s}\right|\right\|_{L^{r}} \leq C\left\|[M, M]_{\infty}^{\frac{1}{2}}\right\|_{L^{q}} .
$$

Hence, for a martingale $M$ in $\mathcal{S}^{q}\left(\mathcal{F}_{t}\right)$ with decomposition $M=(M-[M, L])+$ $[M, L]$ relative to $\left(\mathcal{G}_{t}\right)$, we have 


$$
\begin{aligned}
\|M\|_{\mathcal{S}^{r}\left(\mathcal{G}_{t}\right)} & =\left\|[M, M]_{\infty}^{\frac{1}{2}}+\int_{0}^{\infty}\left|d[M, L]_{s}\right|\right\|_{L^{r}} \\
& \leq\left\|[M, M]_{\infty}^{\frac{1}{2}}\right\|_{L^{r}}+\left\|\int_{0}^{\infty}\left|d[M, L]_{s}\right|\right\|_{L^{r}} \\
& \leq\left\|[M, M]_{\infty}^{\frac{1}{2}}\right\|_{L^{r}}+C\left\|[M, M]_{\infty}^{\frac{1}{2}}\right\|_{L^{q}} \\
& \leq(1+C)\left\|[M, M]_{\infty}^{\frac{1}{2}}\right\|_{L^{q}} \\
& \leq(1+C)\|M\|_{\mathcal{S}^{q}\left(\mathcal{F}_{t}\right)} .
\end{aligned}
$$

Therefore the map $\mathcal{S}^{q}\left(\mathcal{F}_{t}\right) \rightarrow \mathcal{S}^{r}\left(\mathcal{G}_{t}\right), X \mapsto X$, is continuous.

Now suppose the embedding to be continuous. Then Lemma 8 implies

$$
E\left[[\bar{L}, \bar{L}]_{\infty}^{p}\right]<\infty .
$$

So by Lemma 6 the proof is complete.

Example 3. Suppose $\mathcal{A}$ is generated by a countable partition $\mathcal{P}=\left\{A_{1}, A_{2}, \ldots\right\}$ of $\Omega$ into $\mathcal{F}_{\infty}$-measurable sets. Then the corresponding initial enlargement can be viewed as enlargement by the discrete random variable $G(\omega):=$ $\sum_{n} n 1_{A_{n}}(\omega)$. Hence, for $p \geq 1$, we have by Lemma 7

$$
H_{\overline{\mathcal{F}}_{\infty}}^{p}(\bar{P} \| \bar{Q})=\sum_{i \geq 1} P\left(A_{i}\right)\left(\log \frac{1}{P\left(A_{i}\right)}\right)^{p} .
$$

Now let $q, r \geq 0$ such that $\frac{1}{r}=\frac{1}{2 p}+\frac{1}{q}$. Theorem 6 implies that the embedding $\mathcal{S}^{q}\left(\mathcal{F}_{t}\right) \rightarrow \mathcal{S}^{r}\left(\mathcal{G}_{t}\right), X \mapsto X$, is a continuous if and only if

$$
\sum_{i \geq 1} P\left(A_{i}\right)\left(\log \frac{1}{P\left(A_{i}\right)}\right)^{p}<\infty .
$$

This result was already shown by Marc Yor, using different arguments (see Théorème 2 in [Yor85]).

\subsection{Continuity and Shannon Information}

If the filtration $\left(\mathcal{F}_{t}\right)$ is generated by a fixed martingale $M$ with cadlag paths, then the relative entropy of $\bar{P}$ with respect to $\bar{Q}$ is equal to the so-called mutual information between $M$ and the enlarging $\sigma$-algebra $\mathcal{A}$. We recall this notion.

Definition 3. Let $X$ and $Y$ be two random variables with values in the measure spaces $(M, \mathcal{M})$ and $(K, \mathcal{K})$ respectively. The mutual information between $X$ and $Y$ is defined by

$$
I(X, Y)=H_{\mathcal{M} \otimes \mathcal{K}}\left(P_{(X, Y)} \| P_{X} \otimes P_{Y}\right) .
$$

Similarly, one can define the generalized mutual information to be

$$
I^{p}(X, Y)=H_{\mathcal{M} \otimes \mathcal{K}}^{p}\left(P_{(X, Y)} \| P_{X} \otimes P_{Y}\right), \quad p>1 .
$$


For a given $\sigma$-algebra $\mathcal{J} \subset \mathcal{F}$ let $\operatorname{id}_{\mathcal{J}}$ denote the map $(\Omega, \mathcal{F}) \rightarrow(\Omega, \mathcal{J}), \omega \mapsto \omega$. The mutual information between $X$ and $\mathcal{J}$ is defined by

$$
I(X, \mathcal{J})=I\left(X, \operatorname{id}_{\mathcal{J}}\right)
$$

We start with the following observation.

Lemma 9. If $\left(\mathcal{F}_{t}\right)$ equals the filtration generated by $M$, then

$$
I(M, \mathcal{A})=H(\bar{P} \| \bar{Q})
$$

and for $p>1$,

$$
I^{p}(M, \mathcal{A})=H^{p}(\bar{P} \| \bar{Q}) .
$$

Proof. First observe that $\overline{\mathcal{F}}=\mathcal{F}_{\infty} \otimes \mathcal{A}$, because

$$
\overline{\mathcal{F}}=\bigvee_{t} \overline{\mathcal{F}}_{t} \subset \bigvee_{t}\left(\mathcal{F}_{t} \otimes \mathcal{A}\right) \subset \mathcal{F}_{\infty} \otimes \mathcal{A} \subset \overline{\mathcal{F}}
$$

Now let $\mathbb{D}$ denote the Skorokhod space. We define a map $\phi$ by

$$
\Omega \times \Omega \rightarrow \mathbb{D} \times \Omega,\left(\omega, \omega^{\prime}\right) \mapsto\left(M .(\omega), \omega^{\prime}\right) .
$$

Since $\mathcal{F}_{\infty}$ is generated by $M$, we have

$$
\phi^{-1}(\mathcal{B}(\mathbb{D}) \otimes \mathcal{A})=M^{-1}(\mathcal{B}(\mathbb{D})) \otimes \mathcal{A}=\mathcal{F}_{\infty} \otimes \mathcal{A},
$$

and hence

$$
H_{\overline{\mathcal{F}}}(\bar{P} \| \bar{Q})=H_{\mathcal{B}(\mathbb{D}) \otimes \mathcal{A}}\left(\bar{P}_{\phi} \| \bar{Q}_{\phi}\right) .
$$

Now observe

$$
\bar{P}_{\phi}=P_{\phi \circ \psi}=P_{\left(M, \text { id }_{\mathcal{A}}\right)}
$$

and

$$
\bar{Q}_{\phi}=P_{M} \otimes P_{\mathrm{id}_{\mathcal{A}}},
$$

which yields the first claim. The second follows by similar arguments.

As a consequence we obtain the following.

Theorem 7. Suppose assumption $(C)$ is satisfied and let $p \geq 1$ and $q, r \geq 0$ such that $\frac{1}{r}=\frac{1}{2 p}+\frac{1}{q}$. If $\left(\mathcal{F}_{t}\right)$ equals the filtration generated by $M$, then the generalized mutual information $I^{p}(M, \mathcal{A})$ is finite if and only if the embedding

$$
\mathcal{S}^{q}\left(\mathcal{F}_{t}\right) \rightarrow \mathcal{S}^{r}\left(\mathcal{G}_{t}\right), X \mapsto X,
$$

is a continuous linear mapping.

Proof. This follows by combining Theorem 6 with Lemma 9 . 
Example 4. Let $W$ be the standard Wiener process and $\left(\mathcal{F}_{t}\right)$ the filtration generated by $W$ and completed by the negligible sets relative to the Wiener measure. Moreover, let $V$ be a Gaussian element independent of $\mathcal{F}_{\infty}$, with zero mean and variance $w>0$. Suppose the enlarging $\sigma$-algebra $\mathcal{A}$ is generated by the random variable

$$
W_{1}+V
$$

One can easily verify that three random variables $X, Y$ and $Z$ satisfy

$$
I^{p}(X,(Y, Z)) \leq I^{p}(X, Z)+I^{p}(X, Y \mid Z) \quad(p \geq 1) .
$$

Consequently, we obtain for the mutual information between $\operatorname{id}_{\mathcal{A}}$ and $W$

$$
\begin{aligned}
I^{p}\left(W, \operatorname{id}_{\mathcal{A}}\right) & =I^{p}\left(W_{1}+V,\left(W_{1},\left(W_{t}\right)_{0 \leq t<1}\right)\right) \\
& \leq I^{p}\left(W_{1}+V, W_{1}\right)+I^{p}\left(W_{1}+V,\left(W_{t}\right)_{0 \leq t<1} \mid W_{1}\right) \\
& =I^{p}\left(W_{1}, W_{1}+V\right) \\
& <\infty .
\end{aligned}
$$

Thus, for all $p \geq 1$ and $q, r \geq 0$ such that $\frac{1}{r}=\frac{1}{2 p}+\frac{1}{q}$, the mapping $\mathcal{S}^{q}\left(\mathcal{F}_{t}\right) \rightarrow$ $\mathcal{S}^{r}\left(\mathcal{G}_{t}\right), X \mapsto X$, is continuous.

\section{References}

[ADI04] S. Ankirchner, S. Dereich, and P. Imkeller. The Shannon information of filtrations and the additional logarithmic utility of insiders. Preprint, July 2004.

[AIS98] J. Amendinger, P. Imkeller, and M. Schweizer. Additional logarithmic utility of an insider. Stochastic Process. Appl., 75(2):263-286, 1998.

[Ank05] S. Ankirchner. Information and semimartingales. Ph.D. thesis, HumboldtUniversität, 2005.

[DM82] C. Dellacherie and P.A. Meyer. Probabilities and Potential. North Holland, Amsterdam, 1982.

[FI93] H. Föllmer and P. Imkeller. Anticipation cancelled by a Girsanov transformation: a paradox on Wiener space. Ann. Inst. H. Poincaré Probab. Statist., 29(4):569-586, 1993.

[GP98] A. Grorud and M. Pontier. Insider trading in a continuous time market model. International Journal of Theoretical and Applied Finance, 1:331347, 1998.

[GV03] D. Gasbarra and E. Valkeila. Initial enlargement: a Bayesian approach. Preprint, 2003.

[Imk96] P. Imkeller. Enlargement of the Wiener filtration by an absolutely continuous random variable via Malliavin's calculus. Probab. Theory Related Fields, 106(1):105-135, 1996.

[Imk03] P. Imkeller. Malliavin's calculus in insider models: additional utility and free lunches. Math. Finance, 13(1):153-169, 2003. Conference on Applications of Malliavin Calculus in Finance (Rocquencourt, 2001).

[IPW01] P. Imkeller, M. Pontier, and F. Weisz. Free lunch and arbitrage possibilities in a financial market model with an insider. Stochastic Process. Appl., 92(1):103-130, 2001. 
[Jac79] J. Jacod. Calcul stochastique et problèmes de martingales, volume 714 of Lecture Notes in Mathematics. Springer, Berlin, 1979.

[Jac85] J. Jacod. Grossissement initial, hypothese (H'), et théorème de Girsanov. In Th. Jeulin and M. Yor, editors, Grossissements de filtrations: exemples et applications, pages 15-35. Springer-Verlag, 1985.

[JY85] Th. Jeulin and M. Yor, editors. Grossissements de filtrations: exemples et applications, volume 1118 of Lecture Notes in Mathematics. SpringerVerlag, Berlin, 1985. Papers from the seminar on stochastic calculus held at the Université de Paris VI, Paris, 1982/1983.

[LV87] F. Liese and I. Vajda. Convex statistical distances, volume 95 of TeubnerTexte zur Mathematik [Teubner Texts in Mathematics]. BSB B. G. Teubner Verlagsgesellschaft, Leipzig, 1987. With German, French and Russian summaries.

[Pro04] Ph. Protter. Stochastic Integration and Differential Equations. SpringerVerlag, New York, second edition, 2004.

[SY78] C. Stricker and M. Yor. Calcul stochastique dépendant d'un paramètre. $Z$. Wahrsch. Verw. Gebiete, 45(2):109-133, 1978.

[Yoe85] C. Yoeurp. Grossissement de filtration et théorème de girsanov généralisé. In Th. Jeulin and M. Yor, editors, Grossissements de filtrations: exemples et applications. Springer-Verlag, 1985.

[Yor85] M. Yor. Entropie d'une partition, et grossissement initial d'une filtration. In Th. Jeulin and M. Yor, editors, Grossissements de filtrations: exemples et applications, pages 45-58. Springer-Verlag, 1985. 